\title{
Optimized computational techniques-based inventory control process in supply chain management
}

\author{
Hiba A. Tarish \\ Civil Engineering Department, University of Technology, Baghdad, Iraq \\ 120046@uotechnology.edu.iq ${ }^{2}$
}

Article History:Received:11 november 2020; Accepted: 27 December 2020; Published online: 05 April 2021

\begin{abstract}
Now-a-days, supply chain management is one of the crucial factors in business for identifying the flow of goods, raw material storage, movement of products and inventory process so on. The main aim of the supply chain management process is to provide the valuable business services, infrastructure, logistic control and inventory control to the business for improving the business. Among the various supply chain factors, inventory control is one of the difficult tasks in supply chain management process because of high cost inventory, consistent stockouts, low rate of inventory turnover, high value of obsolete inventory, maximum working capital, huge cost for storage and lot customers. Then, several supply chain management techniques are introduced in traditional to control the inventory process but they are failing to detect with greatest accuracy due to the low convergence rate in computation techniques. So, in this paper introduce the effective and optimized neural network computational approach for managing the inventory control. In addition to this, the system ability to select the product based on the few factors that improve the inventory control system accuracy. Then the excellence of the system is evaluated using experimental analysis and respective performance metrics.
\end{abstract}

Keywords: Supply Chain Management, inventory control, business, obsolete inventory, optimized neural network computation techniques and accuracy

\section{Introduction}

Now-a-days most of the business process focuses on the supply chain management (SCM) process [1] because it provides several raw materials, products to the business process with minimum cost but satisfying customer demands successfully. In general, the supply chain process creates the interrelation between the stakeholders and customers in the business process [2]. Depending on the supply chain management professionals, reported that the supply chain management is process of providing the planning, activities related to logistic management process, procurement, sourcing and so on. Among the various business activities, SCM process [3] successfully integrate the service provider and customer for improving the growth of the business. From the different process, inventory is one of the effective supervision processes in supply chain management process that examines the continuous flow of goods at manufacture side and sales point facilities. The inventory management [4] process maintains the complete record of entire product in sale, return and new items which helps to identify the flow of business. When comes to the larger companies, inventory management process is most complex process due to the organization type and size. In the organization, inventory control [5] includes the product raw material, working products, finished products and semi-finished products. The effective management of inventory improves the profit of entire supply chain process because it successfully manages the cost of inventory. This discussed inventory control and management is more important in supply chain management process[6] because of easy, quick satisfaction of customer, continuous production process and prevent the shortage of raw materials.

Even though, the inventory control [7] played a vital role in supply chain management process, it should be handling several inventory control problems. The inventory control process consists of various non-linear model that are difficult to process, quality indicators are difficult to identify, in ability to self-adaptation, consuming more time while collecting data, indirect process of information collection and several uncertainty factors such as transportation condition, time, continuous changes of demands. So, different inventory management techniques [8] are used to manage goods details, handle the customer demands and improve the company profitable. One of the inventory control technique is stock review[9], which is only applicable to the low level or small business process. In this process, stocks are continuously examined according to the future needs which is mostly works according to the manual process consumes more time to minimum customer demands. Another inventory control technique is just in time (JIT) method [10] in which the product is examined according to the customer requirements, demands and user behavior. The JIT technique satisfies the customer demands without considering the quantity of products, risk and problems. But the main disadvantage of the JIT technique leads to create product or service out-of-stock earlier. The next method is ABC analysis[11], in which inventory is managed according to the values and significance, based on this inventory is categorized into three types. In category $\mathrm{A}, \mathrm{ABC}$ process detects the low quantity goods are identified in the high value, category $\mathrm{B}$, moderate 
quantity with moderate value goods are predicted. Finally, in category $\mathrm{C}$, high quantity, low value goods are identified to manage the inventory process. Even though, ABC process effectively evaluates the high value goods, the resources are need to monitored continuously which is very difficult.

Due to the difficulties involved in the conventional inventory control [12] process, the effective and optimized techniques are needed to manage the user requirements in new environment. For attaining these requirement, neural network model is created to examining the control the inventory model. The traditional neural network effectively processes the relationship between the user and their requirement in every input and hidden layer. Even though, the network model [13] should adapt the environment by making the proper training and learning process. During the learning process system may takes time for updating the weights and inventory information. In addition to this, the neural network model easily converging related to the local minimum and slow convergence. For overcoming this issues inventory control system, in this work effective training and inventory model prediction system is created by applying the propagation rule based trained recurrent neural network. The propagation rule- based approach train the user requirements, continuously updates the weights value of the network to reduce the deviation of computation in dynamic environment. Then the incoming new user requirements are effectively handled in supply chain process using the recurrent network. This discussed neural network inventory control process is created using MATLAB tool and the efficiency is examining using performance metrics. Then the rest of the manuscript is organized as follows, section 2 analyze the various research author opinion and work regarding the inventory control process, section 3 discussing about optimized neural network-based inventory control in supply chain management, section 4 evaluate the efficiency of introduced system and concludes in section 5 .

\section{Related Works}

In this section discusses about the various research authors opinion regarding the inventory control process in supply chain management process. Taylor M., Fox C. (2011) [14] creating the effective inventory management process in supply chain using the dynamic Bayesian networks. The introduced approach creates the platform to examining the user requirements for non-specialist manager by creating the software systems. The system successfully predicts the economic orders, quantity of orders and inventory problems are predicted. From the identified information, markov decisions are handled with the help of Bayesian network which reduces the risk factors involved in the supply chain management process.

AsliBoru et al.,[15] introducing the optimized artificial intelligent techniques to resolve the inventory routing problem. During this process, three different patterns such as lumpy, erratic and intermittent are examined in two phases. In first phase, the genetic algorithm is applied to the user requirements, order level and customer demands are examined with minimum cost of supply chain management process. From the examined business and customer demands, it is processed with the help of neural network to predict the reorder point, customer demand forecast to improve the inventory process. The efficiency of the system is evaluated using the experimental analysis, then, the neural network- based inventory control process ensures the $98.54 \%$ of accuracy and improve the service level in supply chain management process.

TamásVargaet a., [16] resolving the inventory management problems in supply chain process using the particle swarm optimized Monte Carlo Simulation process. Initially, the user requirements and demands are collected which is processed in terms of memory- based algorithm. Then the effective former function is used to predict the complexity involved in the supply chain and the excellence of the system is evaluated using the benchmark of dataset, in which gradient-based particle swarm optimization approach attains the maximum results while managing the inventory problems in supply chain process.

Elkhechafi, $\mathrm{M}$ et al., [17] solving the discrete optimization issue in supply chain management process using firefly algorithm. The collected business-related information is processed by the firefly algorithm which successfully identify the available stocks related to customer needs. During this examination process, cost and level of services related issues are controlled using characteristics of firefly algorithm. The efficiency of the system is determined using experimental results in which the system ensures the better solution to the supply chain management problem comparted to the deterministic approach. According to the above research author opinions, inventory control is one of the main issues in supply chain management process. Depending on their analysis, artificial intelligent techniques are mostly used in the business environment for resolving the computational issues. From the research author opinion, in this work, effective and optimized neural network is used to examine the customer demands on specific business successfully. In addition to this, the introduced method reduces the complexity due to the effective training process. Then the detailed study of inventory control in supply chain management process is discussed in section 3.

\section{Optimized Neural Network based Inventory Control process in supply chain Management}

In this section discusses about the inventory control process[18] in supply chain management process which is done by using the optimized neural network approach. This created system used to examines the inventory level of the company or business using the optimized neural network. During the implementation process, system 
gathers the input from the previous process which includes the wholesale retailer information, supplier of the company, part of supply chain details, delivery time, date of order, current demand level, demand in after 3 months, 3-month order cycle demands, current inventory level, purchasing price and transport cost information [19]. The collected business requirements are processed by the intelligent artificial technique to determine the inventory details effectively. First the collected information is arranged in the working space, if the collected information having any missing data, it should be replaced by the computation of average of specific details. After replacing the missing values, it is processed by the propagation rule based trained recurrent neural network. Initially, the training model is created using the propagation approach because it effective maintains the inventory information [20] by doing continuous updating of network weight value.

\section{Propagation based Training process}

Before making the training process, inventory model should be created. In this work, automotive Manufacturer Service Operation Management (MSOM) publicly available business dataset [21] information is used to analyze the inventory control process in supply chain management process. During this process, system should consider that supplier level information, wholesales, manufacturers and retailer details. After collecting the details, here discussing the few factors [22] that influencing the inventory control process. First different cost factor is analyzed because, cost only determine the purchasing and production of the business product. The effective control of inventory cost leads to reduces the overall enterprises cost because, the cost includes in shortage cost, transportation cost and storage cost. Second factor is demand level, in which, business process should satisfy the demands of the customer because the demand is proportional to the inventory level of the business. Third factor is supply level; it indicates that the finished products that is proportional to business inventory level. Next factor is quantity of substitutes, which is used to substitute of other part, that is negatively proportional to business inventory level. Lead time is another factor of inventory level, which examines the product order time, waiting time, manufacturing time, supplier delivery time, transportation time, quality checking time and usage time. So, the time is positively proportional to the business inventory level.Next factor is customer service level, which refers to the satisfaction of customer level for ordering or receiving the products with respect to their demands. The customer service level is negatively proportional to the business inventory level. Based on the above business factors, the data is collected from the company which is analyzed using the introduced computational techniques [23]. First the gathered data is divided into two parts such as training (80\%) and testing (20\%) in which the training part is high because, the system must be learn and adapt to the inventory control management system. During the training process system able to analyze different type of business data which helps when the new incoming customer demands easily. As discussed earlier, before making the training process, missing value should be removed and the data should be normalized to change the representation of the data into 0 to 1 . After that the training process is performed with the help of propagation chain rule in which errors [24] are propagated in forward and backward to get the effective neural structure. During the training process error offset process is added in the network for resolving the convergence problem in propagation training process. The training network consists of three layers such as input, hidden and output layer. As discussed in the inventory factors, the input layer receives 9 inputs such as ordering cost, storage cost, transportation cost, shortage cost, demand level, quantity substitutes, supply level, service level and waiting time. The incoming input is processed by the hidden layer in this work 3 hidden layers are used to process the given non-linear inputs and produce the linear output effectively. During the training process, the error [25] must be computed to get the exact value. So, the error value must be defined while train the network which is done as follows,

$$
\text { Error }=\frac{1}{2} \sum_{p}\left(a^{p}-y^{p}\right)^{2}
$$

In eqn (1) a is the target output of pth sample in training, $y$ is the actual output of $p$ th sample in training. The obtained error value is redefined for reducing the convergence rate.

$$
\text { Redefined Error }=\frac{1}{2} \sum_{p}\left(a^{p}-y^{p}\right)^{2}+\frac{1}{2} \sum_{p}\left(f^{-1}\left(a^{p}\right)-f^{-1}\left(y^{p}\right)\right)^{2}
$$

Here, $\frac{1}{2} \sum_{p}\left(f^{-1}\left(a^{p}\right)-f^{-1}\left(y^{p}\right)\right)^{2}$ is denoted as error offset value of training process.

After redefining the error value, the weight value of neural network [26] must be computed which is done using eqn (3) that is delta rule.

$$
w_{i, j}^{m}(p+1)=w_{i, j}^{m}(p)+\Delta w_{i, j}^{m}
$$

In eqn (3), $\mathrm{m}$ is the neural network layer, $\mathrm{w}$ is weight value. Then the $\Delta w_{i, j}^{m}$ is computed as follows,

$$
\Delta w_{i, j}^{m}=-\eta \frac{\partial \text { error }}{\partial w_{i, j}}
$$

Here learning rate is represented as the $\eta$. According to the redefined error value, the new weight value is estimated as follows

$$
w_{i, j}^{m}(p+1)=w_{i, j}^{m}(p)+\eta\left[(a-y) * f\left(I_{j}\right) * O_{j}+\left(f^{-1}\left(a_{j}\right)-I_{j}\right) O_{j}\right]
$$

During the learning process, the network reaches the output node, then the weight computation is done as follows. 


$$
\frac{\partial \text { error }}{\partial w_{i, j}}=-(a-y) * f\left(I_{j}\right) * O_{j}
$$

Else, the still the network having the hidden layers, the error propagation is still working as eqn (7)

$$
w_{i, j}^{m}(p+1)=w_{i, j}^{m}(p)+\eta\left[f\left(I_{j}\right) \sum_{m}\left(a^{k}-y^{k}\right) f\left(I_{m}\right) w_{m j}+\left(f^{-1}\left(a_{j}^{p}\right)-I_{k}\right) \sum_{m} f\left(I_{j}\right) w_{m j} O_{i}\right] 7
$$

Based on this process, the training model is successfully created, which helps to examine the new incoming business needs in supply chain management.

\section{Inventory control handling using recurrent neural network}

The inventory control is handled when the new business requirement coming into the system which is analyzed by using the recurrent network [27]. The network examines the new features, according to the directed graph, because, which consists of sequence of link between one business needs to another. So, the customer behavior on specific needs are effectively evaluated in dynamic manner. During the analysis process, the internal state is used to process the incoming business inputs. As discussed earlier, it is three-layer network with context unit. The middle-hidden layer context unit is fixed weight value while computing the output. The incoming input is processed by using this defined context unit with learning rule. The computed value is forwarded to the next layer for computing the output value. Then the output computation of hidden layer [28] is defined as follows.

$$
h_{t}=\sigma_{h}\left(W_{h} x_{t}+U_{h} h_{t-1}+b_{h}\right)
$$

In eqn (8) $x_{t}$ is input vector in neural network, $h_{t}$ is hidden layer, $\mathrm{W}, \mathrm{u}$ and $\mathrm{b}$ is parameter utilized in network and $\sigma_{h}$ is activation function. In this work, sigmoid activation function is used to get the output in each layer. The activation function is done as follows,

$$
\sigma_{h}=\frac{1}{1+e^{-x}}
$$

Then the final output is estimated as follows,

$$
y_{t}=\sigma_{y}\left(W_{y} h_{t}+b_{y}\right)
$$

In eqn (9), y is output value. And the same sigmoid function is used for the activation function.

The successful training process helps to predict the output to the customer demands. Based on the output inventory has been managed and controlled in supply chain process. Then the efficiency of the system is evaluated using experimental analysis.

\section{Results and Discussion}

In this section discusses about the excellence of Optimized Neural Network based Inventory Control process in supply chain Management. During the development process system uses the Manufacturer Service Operation Management (MSOM) publicly available business dataset. From the collected data, $80 \%$ of details are used to train with the help of propagated error offset approach. Then $20 \%$ of used for testing purpose which used to predict the how effectively system reacts on the new incoming customer request in supply chain management process. Based on the discussion, the system is developed using MATLAB tool, in which excellence of the system is determined using the error rate, prediction accuracy and time effect on inventory control system. The error value of the system is estimated as follows.

$$
\begin{aligned}
& \text { Absolute Error Value }(A E V)=\left|\frac{\hat{y}_{i}-y_{i}}{y_{i}}\right| * 100 \\
& \text { Mean Absolute Error Value }(M A E V)=\frac{1}{n} \cdot \sum_{i=1}^{n}\left|\frac{\hat{y}_{i}-y_{i}}{y_{i}}\right| * 100
\end{aligned}
$$

In eqn (11 and 12) y is the estimated output value and $\hat{y}_{i}$ is the actual output value. According to the computation, the obtained result is demonstrated in table 1

Table 1: Error rate

\begin{tabular}{|l|r|r|}
\hline & $\begin{array}{l}\text { Mean Absolute } \\
\text { Cosolute Error } \\
\text { Value (AEV) }\end{array}$ & $\begin{array}{l}\text { Error Value } \\
\text { (MAEV) }\end{array}$ \\
\hline Dynamic Bayesian Networks (DBN) & 0.124 & 0.11 \\
\hline Artificial Neural Networks (ANN) & 0.102 & 0.097 \\
\hline $\begin{array}{l}\text { Gradient Particle Swarm Optimization } \\
\text { Networks (GPSO) }\end{array}$ & 0.098 & 0.0783 \\
\hline $\begin{array}{l}\text { Fireflies artificial neural network (FA- } \\
\text { ANN) }\end{array}$ & 0.076 & 0.065 \\
\hline $\begin{array}{l}\text { optimized error propagated recurrent neural } \\
\text { network (OERNN) }\end{array}$ & 0.0067 & 0.00546 \\
\hline
\end{tabular}

The above table 1 value is computed from the eqn (11) and (12), the deviation of $\hat{y}_{i}$ and y is very low due to the effective pre-training model. The training model uses the optimized $\Delta w_{i, j}^{m}$ value while the system ensures the error. From the analysis, OERNN attains minimum error rate compared to the other computation techniques. Based on the discussion, the estimated error related graphical representation is depicted in figure 1. 


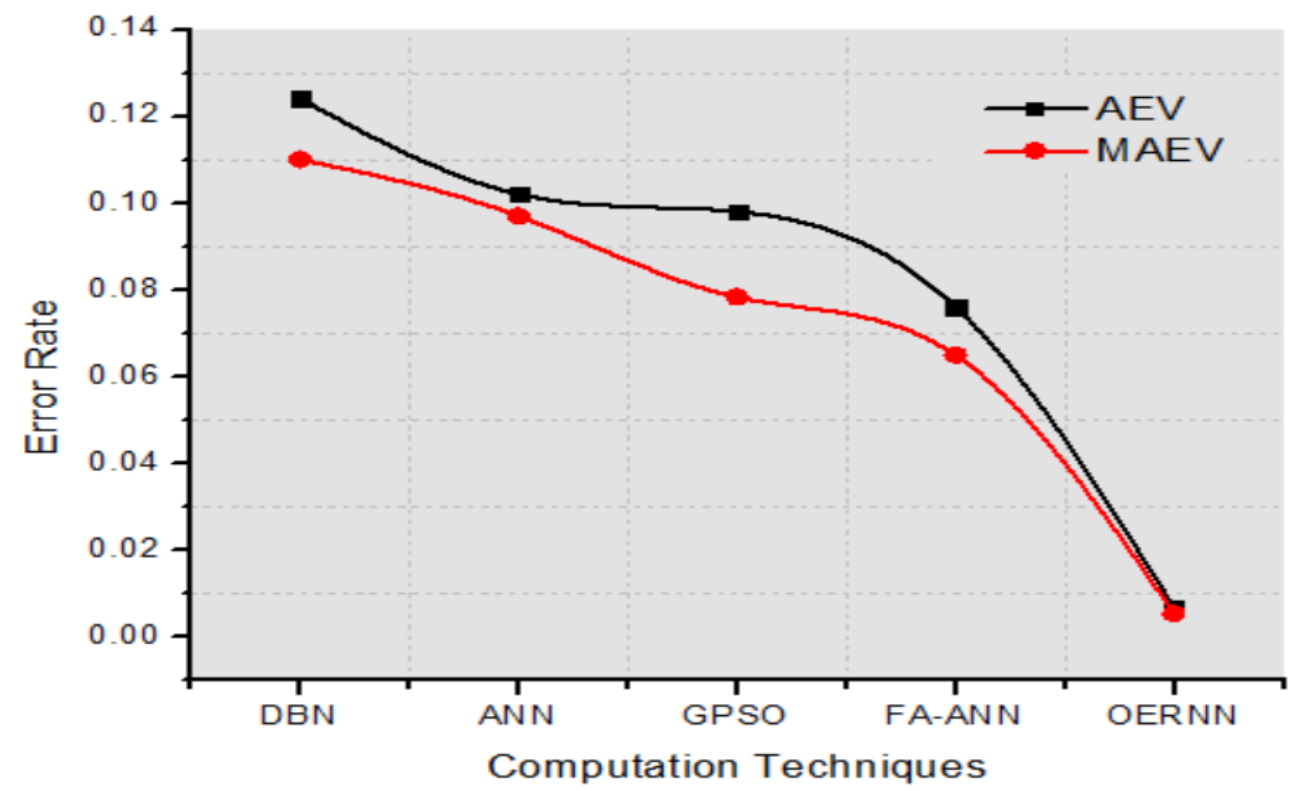

Figure 1: Error Rate

From the figure 1, depicted that the optimized error propagated recurrent neural network (OERNN) approach attains minimum error rate while controlling the inventory details in supply chain management process. Due to the effective propagation of error values in every hidden layers in network leads to reduces the deviation of errors [30-35]. In addition to this, the system uses the successful training process that effectively handles the large volume user requirements and control the inventory issues in supply chain management process. The system ensures minimum error value (AEV-0.0067, MAEV-0.00546) compared to other methods such as dynamic Bayesian network (DBN)(AEV-0.124, MAEV-0.11), artificial neural network (ANN)(AEV-0.102, MAEV-0.097), gradient particle swarm optimization network (GPSO) (AEV-0.098, MAEV-0.0783) and fireflies algorithm neural network (FA-ANN)(AEV-0.076, MAEV-0.065). The minimum error rate leads to maximize the overall inventory control prediction accuracy which is measured in terms of precision, recall and accuracy metrics. From the analysis, the obtained result is depicted in table 2.

Table 2: Efficiency

\begin{tabular}{|l|r|r|r|}
\hline Computational Techniques & \multicolumn{1}{|c|}{ Precision } & \multicolumn{1}{c|}{ Recall } & \multicolumn{1}{c|}{ Accuracy } \\
\hline Dynamic Bayesian Networks (DBN) & 98.42 & 98.43 & 98.425 \\
\hline Artificial Neural Networks (ANN) & 98.41 & 98.53 & 98.47 \\
\hline $\begin{array}{l}\text { Gradient Particle Swarm Optimization } \\
\text { Networks (GPSO) }\end{array}$ & 98.67 & 98.76 & 98.715 \\
\hline $\begin{array}{l}\text { Fireflies Artificial Neural Network (FA- } \\
\text { ANN) }\end{array}$ & 98.87 & 98.54 & 98.605 \\
\hline $\begin{array}{l}\text { optimized error propagated recurrent } \\
\text { neural network (OERNN) }\end{array}$ & 99.234 & 99.21 & 99.222 \\
\hline
\end{tabular}

Table 2 demonstrates that the efficiency value of optimized error propagated recurrent neural network (OERNN) approach. The system computes the output according to $y_{t}=\sigma_{y}\left(W_{y} h_{t}+b_{y}\right)$ in which the weight value is effectively replaced by optimized $\Delta w_{i, j}^{m}$ value. During the output estimation process, the trained inventory features are more helpful to predict the exact value of the incoming new input details. Due to these reason, OERNN approach attains the maximum accuracy compared to other computational techniques. Then the relevant graphical analysis is demonstrated in figure 2 . 


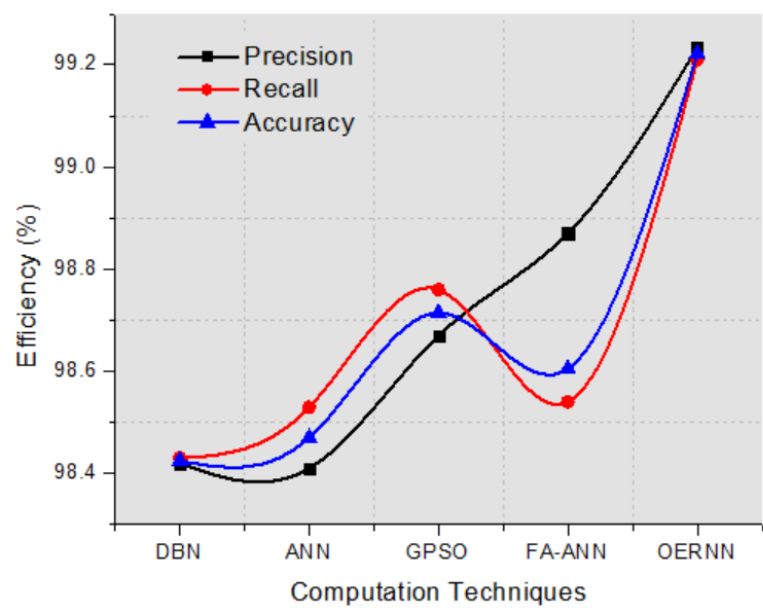

Figure 2: Prediction Efficiency

The figure 2 demonstrated that the excellence of inventory control prediction efficiency which is measured in terms of using precision, recall and accuracy. Among the other computation techniques such as dynamic Bayesian network (DBN)(precision-98.42\%, recall-98.43\% accuracy-98.425\%), artificial neural network (ANN)(precision-98.41\%, recall-98.53\% accuracy-98.47\%), gradient particle swarm optimization network (GPSO) (precision-98.672\%, recall-98.76\% accuracy-98.71\%) and fireflies algorithm neural network (FAANN)(precision-98.87\%, recall-98.94\% accuracy-98.90\%) compared to optimized error propagated recurrent neural network (OERNN)(precision-99.23\%, recall-99.21\% accuracy-99.25\% attains maximum inventory control prediction accuracy. Due to the effective utilization of learning rule, weight updating process, error propagation leads to maximize the user demands and inventory control examination process. In addition to this, prediction accuracy, the effectiveness of the system is determined using the inventory control prediction time in both testing and training process. The respective time analyzed value is shown in table 3 .

Table 3: Time Analysis

\begin{tabular}{|l|r|r|}
\hline Computational Techniques & \multicolumn{1}{l|}{$\begin{array}{l}\text { Training } \\
\text { Time }\end{array}$} & $\begin{array}{l}\text { Testing } \\
\text { Time }\end{array}$ \\
\hline Dynamic Bayesian Networks (DBN) & 12.34 & 11.8 \\
\hline Artificial Neural Networks (ANN) & 11.79 & 10.34 \\
\hline Gradient Particle Swarm Optimization Networks (GPSO) & 10.34 & 9.46 \\
\hline $\begin{array}{l}\text { optimized error propagated recurrent neural network } \\
\text { (OERNN) }\end{array}$ & 9.68 & 8.45 \\
\hline Fireflies Artificial Neural Networks (FA-ANN) & 8.367 & 6.78 \\
\hline OERNN & 12.34 & 11.8 \\
\hline
\end{tabular}

The system uses the effective learning function which train the supply chain related features with minimum time. Due to the effective training process, system helps to recognize the new incoming features related inventory information with minimum time. Then the relevant graphical analysis is depicted in figure 3 .

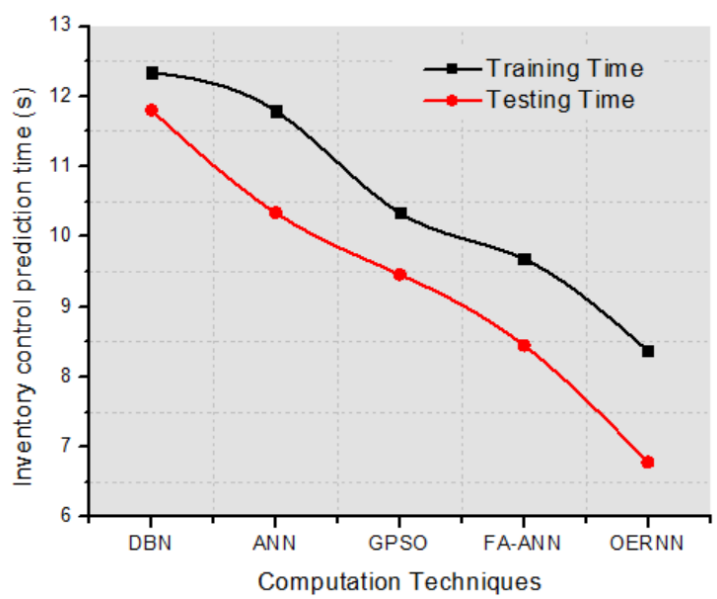

Figure 3: Inventory control prediction time 
As discussed in section 3, the optimized error propagated recurrent neural network (OERNN) uses $80 \%$ of data to the training process. Due to the huge volume of trained data helps to make the system according to the inventory control prediction process. In addition to this, the system ability to propagate the error and detect the inventories by using effective learning rule. So, the the optimized error propagated recurrent neural network (OERNN) (7.57s) approach attains the minimum computation time compared to other approaches such asdynamic Bayesian network (DBN)(12.07s), artificial neural network (ANN)(11.06s), gradient particle swarm optimization network (GPSO) (9.9s) and fireflies algorithm neural network (FA-ANN)(9.06s). In addition to this, the ROC (receiver operating characteristics) curve examination of the inventory control is examined and the respective analysis depicted in figure 4.

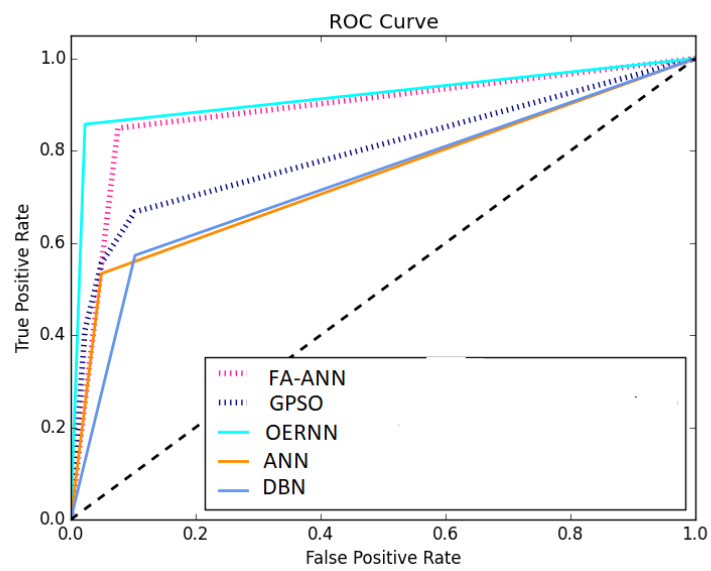

Figure 4: ROC Analysis

From the figure 4, it clearly demonstrates that the OERNN approach attains maximum ROC value which the introduced OERNN method effectively control inventory in supply chain process compared to other methods. The system uses the error propagated delta rule concept which reduce the deviation of features also classification is done by using the vector units and hidden units. These defined units effectively recognize the consumer needs related inventories successfully. Thus the introduced optimized error propagated recurrent neural network (OERNN) effectively controls the inventory in supply chain management process.

\section{Conclusion}

Thus, the paper analyzes the optimized error propagated recurrent neural network (OERNN) based inventory control process in supply chain management process. Initially, the business data is collected from the Manufacturer Service Operation Management (MSOM) publicly available business dataset. The dataset is processed by average computation technique to remove the missing value in the dataset. After that the data representation is changed to 0 to 1 and the divide the dataset into $80 \%$ of training and $20 \%$ testing purpose. Then the training model is created by propagating the error into the previous layer while network handles any deviation of computed and target value. This process is repeated in three-layer of neural network. The created training process is depends on the several business requirement such as cost, service level, demand, production and so on. Then the new incoming features are arrived into the system, the requirements are processed according to the recurrent network indirect graph representation process which successfully derives the output of the specific input. Finally, the excellence of the system is determined using the MATLAB results in which system ensures minimum error rate (0.00564), maximum inventory prediction accuracy $(99.22 \%)$ with minimum time $(7.57 \mathrm{~s})$ while controlling inventories in supply chain management process.

\section{Reference}

Lavastre O, Gunasekaran A, Spalanzani A (2014) Effect of firm characteristics, supplier relationships and techniques used on supply chain risk management (SCRM): an empirical investigation on French industrial firms. Int J Prod Res 52(11):3381-3403. doi: 10.1080/00207543.2013.878057

Hou C-K (2012) Examining the effect of user satisfaction on system usage and individual performance with business intelligence systems: an empirical study of Taiwan's electronics industry. Int J Inf Manage 32(6):560-573. doi: 10.1016/j.ijinfomgt.2012.03.001

Kotzab H, Teller C, Grant Db, Sparks L (2011) Antecedents for the adoption and execution of supply chain management. Supply Chain ManagInt J 16(4):231-245.

X. P. Wang, Y. Shi, J. B. Ruan, and H. Y. Shang, "Study on the inventory forecasting in supply chains based on rough set theory and improved BP neural network," in Advances in Intelligent Decision Technologies Smart Innovation, Systems and Technologies, vol. 4, pp. 215-225, Springer, Berlin, Germany, 2010. 
K. Zhang, J. Xu, and J. Zhang, “A new adaptive inventory control method for supply chains with non-stationary demand," in Proceedings of the 25th Control and Decision Conference (CCDC '13), pp. 1034-1038, Guiyang , China, May 2013

Pradabwong, J., Braziotis, C., Pawar, K.S. et al. , "Business process management and supply chain collaboration: a critical comparison", Logist. Res. (2015) 8: 6. https://doi.org/10.1007/s12159-015-0123-6.

Dolgui A, Pashkevich M (2008) On the performance of binomial and beta-binomial models of demand forecasting for multiple slow-moving inventory items. Comput. Oper. Res. 35(3):893-905

S. Minner, "Multiple-supplier inventory models in supply chain management: a review," International Journal of Production Economics, vol. 81-82, pp. 265-279, 2003

Olsson, F. , "Simple modeling techniques for base-stock inventory systems with state dependent demand rates ", Math Meth Oper Res (2019) 90: 61. https://doi.org/10.1007/s00186-018-0654-0

Qureshi, M.I., Iftikhar, M., Bhatti, M.N. et al. , "Critical elements in implementations of just-in-time management: empirical study of cement industry in Pakistan ", SpringerPlus (2013) 2: 645. https://doi.org/10.1186/2193-1801-2-645

Chen Y., Li K.W., Levy J., Hipel K.W., Kilgour D.M. (2008) A Rough Set Approach to Multiple Criteria ABC Analysis. In: Peters J.F., Skowron A. (eds) Transactions on Rough Sets VIII. Lecture Notes in Computer Science, vol 5084. Springer, Berlin, Heidelberg

S. R. Hong, S. T. Kim, and C. O. Kim, "Neural network controller with on-line inventory feedback data in RFID-enabled supply chain," International Journal of Production Research, vol. 48, no. 9, pp. 2613-2632, 2010

Memari A., Rahim A.R.B.A., Ahmad R.B. (2014) Production Planning and Inventory Control in Automotive Supply Chain Networks. In: Ali M., Pan JS., Chen SM., Horng MF. (eds) Modern Advances in Applied Intelligence. IEA/AIE 2014. Lecture Notes in Computer Science, vol 8481. Springer, Cham

Taylor M., Fox C. (2011) Inventory Management with Dynamic Bayesian Network Software Systems. In: Abramowicz W. (eds) Business Information Systems. BIS 2011. Lecture Notes in Business Information Processing, vol 87. Springer, Berlin, Heidelberg

AslıBoru, Ay,seTu ־gbaDosdo ־gru, Mustafa Göçken and RızvanErol, “A Novel Hybrid Artificial Intelligence Based Methodology for the Inventory Routing Problem", Symmetry 2019, 11, 717; doi:10.3390/sym11050717.

TamásVarga, AndrásKirály, JánosAbonyi, "Improvement of PSO algorithm by memory based gradient search application in inventory management",https://arxiv.org/ftp/arxiv/papers/1410/1410.5652.pdf

Elkhechafi, M., Benmamoun, Z., Hachimi, H. et al. Lobachevskii, " Firefly Algorithm for Supply Chain Optimization “, J Math (2018) 39: 355. https://doi.org/10.1134/S1995080218030125

$\mathrm{He}$, W. An inventory controlled supply chain model based on improved BP neural network. Internation- al Journal of Industrial Engineering Computations. 2013;2013:1-7. DOI: 10.1155/2013/537675

Paul, S. K., Azaeem, A. An artificial neural network model for optimization of finished goods inventory. International Journal of Industrial Engineering Computations. 2011;2(2):431-438. DOI: 10.5267/ j.ijiec.2011.01.005

Sarkar, B., Sarkar, S. An improved inventory model with partial backlogging, time varying deteriora- tion and stock-dependent demand. Economic Modelling. 2013;30:924-932

https://pubsonline.informs.org/journal/msom

Wu, J., Al-khateeb, F. B., Teng, J. T., CárdenasBarrón, L. E. Inventory models for deteriorating items with maximum lifetime under downstream partial trade credits to credit-risk customers by discounted cash-flow analysis. International Journal of Production Economics. 2016;171(1): 105-115. DOI: 10.1016/j.ijpe.2015.10.020

B. ALhayani, and H. Ilhan, "Efficient cooperative imge transmission in one-Way mult-hop sensor network," International Journal of Electrical Engineering Education, vol.57, no.2, pp.321-339. 2020.

Nawi N.M., Ransing R.S., Salleh M.N.M., Ghazali R., Hamid N.A. (2010) An Improved Back Propagation Neural Network Algorithm on Classification Problems. In: Zhang Y., Cuzzocrea A., Ma J., Chung K., Arslan T., Song X. (eds) Database Theory and Application, Bio-Science and Bio-Technology. BSBT 2010, DTA 2010. Communications in Computer and Information Science, vol 118. Springer, Berlin, Heidelberg

BSA. Al Hayani and H. Ilhan, "Visual Sensor Intelligent Module Based Image Transmission in Industrial Manufacturing for Monitoring and Manipulation problems," Journal of Intelligent Manufacturing, 32, pages597-610(2021)

B. Alhayani, A.A. Abdallah, "Manufacturing intelligent Corvus corone module for a secured two way image transmission under WSN", Engineering Computations, Vol. 37 No. 9, pp. 1-17. 2020

Sridhar KP, Baskar S, Shakeel PM, Dhulipala VS., "Developing brain abnormality recognize system using multi-objective pattern producing neural network", Journal of Ambient Intelligence and Humanized Computing, 2018:1-9. https://doi.org/10.1007/s12652-018-1058-y 
Chung, Junyoung; Gulcehre, Caglar; Cho, KyungHyun; Bengio, Yoshua (2014). "Empirical Evaluation of Gated Recurrent Neural Networks on Sequence Modeling". arXiv:1412.3555

Li, Xiangang; Wu, Xihong (2014-10-15). "Constructing Long Short-Term Memory based Deep Recurrent Neural Networks for Large Vocabulary Speech Recognition". arXiv:1410.4281.

Mahajan, H.B., Badarla, A. \& Junnarkar, A.A. (2020). CL-IoT: cross-layer Internet of Things protocol for intelligent manufacturing of smart farming. $J$ Ambient Intell Human Comput. https://doi.org/10.1007/s12652-020-02502-0.

Mahajan, H.B., \& Badarla, A. (2018). Application of Internet of Things for Smart Precision Farming: Solutions and Challenges. International Journal of Advanced Science and Technology, Vol. Dec. 2018, PP. 37-45.

Mahajan, H.B., \& Badarla, A. (2019). Experimental Analysis of Recent Clustering Algorithms for Wireless Sensor Network: Application of IoT based Smart Precision Farming. Jour of Adv Research in Dynamical \& Control Systems, Vol. 11, No. 9. 10.5373/JARDCS/V11I9/20193162.

Mahajan, H.B., \& Badarla, A. (2020). Detecting HTTP Vulnerabilities in IoT-based Precision Farming Connected with Cloud Environment using Artificial Intelligence. International Journal of Advanced Science and Technology, Vol. 29, No. 3, pp. 214 - 226.

Mikhail, A., Kamil, I. A., \& Mahajan, H. (2017). Increasing SCADA System Availability by Fault Tolerance Techniques. 2017 International Conference on Computing, Communication, Control and Automation (ICCUBEA). doi:10.1109/iccubea.2017.8463911

Mikhail, A., Kareem, H. H., \& Mahajan, H. (2017). Fault Tolerance to Balance for Messaging Layers in Communication Society. 2017 International Conference on Computing, Communication, Control and Automation (ICCUBEA). doi:10.1109/iccubea.2017.8463871 